\title{
Foreskin T-cell subsets differ substantially from blood with respect to HIV co-receptor expression, inflammatory profile, and memory status
}

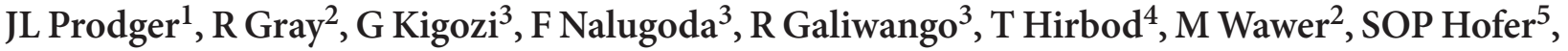 \\ N Sewankambo ${ }^{6}$, D Serwadda ${ }^{7}$ and $\mathrm{R} \mathrm{Kaul}^{1}$
}

The foreskin is the main site of heterosexual human immunodeficiency virus (HIV) acquisition in uncircumcised men, but functional data regarding T-cell subsets present at this site are lacking. Foreskin tissue and blood were obtained from Ugandan men undergoing elective adult circumcision. Tissue was treated by mechanical and enzymatic digestion followed by T-cell subset identification and assessment of cytokine production using flow cytometry. Foreskin CD4 ${ }^{+}$ T cells were predominantly an effector memory phenotype, and compared with blood they displayed a higher frequency of CCR5 expression ( $42.0 \%$ vs. $9.9 \%$ ) and interleukin-17 production. There was no difference in T-regulatory cell frequency, but interferon- $\gamma$ and tumor necrosis factor- $\alpha$ production were increased in foreskin CD8 ${ }^{+} \mathrm{T}$ cells. These novel techniques demonstrate that the foreskin represents a proinflammatory milieu that is enriched for HIV-susceptible T-cell subsets. Further characterization of foreskin T-cell subsets may help to define the correlates of HIV susceptibility in the foreskin.

\section{INTRODUCTION}

As of 2009 there were 33.3 million people infected with human immunodeficiency virus (HIV)-1, and only a third of those requiring treatment were receiving it. ${ }^{1}$ In addition, there were an estimated 2.6 million new infections in that year, the majority transmitted through heterosexual sex, emphasizing the urgent need for better HIV prevention strategies. Clinical trials have demonstrated that circumcision reduces HIV acquisition by $50-60 \%$ in heterosexual men, proving that the foreskin is the site of most acquisition in uncircumcised men exposed to HIV during insertive vaginal sex. ${ }^{2-4}$ Although other penile sites such as the urethra may also have a role, ${ }^{5}$ the central role of the foreskin in HIV acquisition was further supported by the observation that an increased foreskin surface area correlated with increased risk of HIV acquisition. ${ }^{6}$ However, the immune events that surround acquisition and establishment of productive infection in the foreskin are poorly defined. ${ }^{7}$ Understanding the immunopathogenesis of HIV acquisition in the foreskin remains an important priority for the development of new prevention modalities, despite the efficacy of male circumcision, as evidenced by the fact that only a third of eligible men opted to avail themselves of free male circumcision during a recent HIV vaccine trial in South Africa. ${ }^{8}$

In the cervix, HIV and SIV infection is initiated by a small founder population of infected $\mathrm{CD} 4{ }^{+} \mathrm{T}$ cells that expands through the local production of chemoattractant cytokines, followed by subsequent recruitment of activated memory CD $4^{+}$ T cells. ${ }^{9,10}$ It is likely that the efficiency with which this founder virus population expands depends on the immune milieu in the genital mucosa at the time of exposure to HIV. ${ }^{11}$ While resting $\mathrm{CD} 4^{+} \mathrm{T}$ cells can be infected, viral replication within such cells is less efficient, and HIV propagation and dissemination from the site of initial infection are driven by the rapid recruitment of activated $\mathrm{CD} 4^{+} \mathrm{T}$ cells, in which the virus can more readily replicate. ${ }^{9,12}$ Recruitment of these activated $\mathrm{CD} 4^{+} \mathrm{T}$ cells to the initial site of exposure may be assisted by HIV-induced changes in the local immune milieu, including the expression of chemokines such as MIP- $3 \alpha$ and MIP- $1 \beta$ by epithelial and plasmacytoid dendritic cells. ${ }^{9,13}$

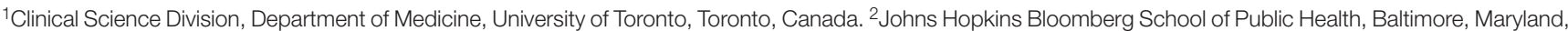
USA. ${ }^{3}$ Rakai Health Sciences Program, Kalisizo, Uganda. ${ }^{4}$ Department of Medicine, Solna, Center for Molecular Medicine, Karolinska Institutet, Stockholm, Sweden. ${ }^{5}$ Department of Surgery, University of Toronto, Toronto, Canada. ${ }^{6}$ College of Health Sciences, Makerere University, Kampala, Uganda. ${ }^{7}$ Institute of Public Health, Makerere University, Kampala, Uganda. Correspondence: R Kaul (rupert.kaul@utoronto.ca) 
The presence or absence of certain T-cell subsets at the mucosal site of HIV exposure may be an important determinant of HIV susceptibility. Genital herpes is associated with an increase in activated $\mathrm{CD} 4^{+} \mathrm{T}$ cells within the foreskin and female genital tract, ${ }^{14-16}$ perhaps contributing to the threefold increase in HIV susceptibility associated with this infection. ${ }^{17}$ The proinflammatory Th 17 cells that normally protect skin and mucosal sites against bacterial and fungal infection are present at high frequency in the female genital mucosa and display enhanced HIV susceptibility. ${ }^{18,19}$ Interleukin (IL)22 is an important effector molecule of Th17 cells, having a role in epithelial integrity and repair. IL-22 is also produced by proinflammatory Th2 2 cells, which may be preferentially infected by HIV ${ }^{20}$ Conversely, CD $25^{+} / \mathrm{FoxP}^{+}$(Forkhead box P3) T-regulatory cells (Tregs) have an important role in controlling inflammation, and higher Treg frequencies in the blood have been linked to reduced HIV susceptibility. ${ }^{21}$ Furthermore, individuals who are HIV exposed but seronegative show a quiescent immune phenotype with reduced basal T-cell cytokine production and lower proportions of activated T cells. ${ }^{21-24}$

Although immunohistochemistry is able to demonstrate the tissue position of specific cells in three dimensions, the ability of this technique to define cellular immune function is very limited. Therefore, we have developed techniques to isolate a single-cell suspension from fresh foreskin tissues, and to characterize the frequency and function of foreskin T-cell subsets using multiparameter flow cytometry. Our results indicate that the foreskin constitutes a proinflammatory immune environment that is enriched for HIV-susceptible T-cell subsets.

\section{RESULTS}

\section{Study population}

Participants were recruited from a longstanding community cohort in Rakai, Uganda. ${ }^{25}$ Foreskin and whole blood were collected from 46 men between the ages 15 and 49 years, who had requested elective circumcision at the Rakai Health Sciences Program clinic in Kalisizo, Uganda, and who had provided written informed consent. All men were free of symptomatic sexually transmitted diseases at the time of surgery.

\section{T-cell proportions in the blood and foreskin}

Foreskin $\mathrm{T}$ cells were identified based on the expression of $\mathrm{CD} 3$, and comprised between 0.1 and $0.6 \%$ of total recorded events from digested, filtered foreskin tissue (Supplementary Material online). Upon permeabilization, many contaminating events (non- $\mathrm{CD}^{+}{ }^{+}$) were removed from the cell solution, so that $\mathrm{CD}^{+}$events constituted $10-15 \%$ of total recorded events, allowing for easier identification of lymphocytes based on forward and side scatter alone. Owing to differences in the size of foreskin samples and to variation in tissue physical properties leading to differential cell loss during the digestion procedure, the absolute number of $\mathrm{CD} 3$ cells per foreskin was not defined. Rather, we report proportions of cells, standardized to $\mathrm{CD}^{+}$, $\mathrm{CD} 3 / 4^{+}$, or $\mathrm{CD} 3 / 8^{+}$. The majority of foreskin $\mathrm{CD} 3^{+}$cells were found to express either $\mathrm{CD} 4$ (mean, $51.4 \%$ of $\mathrm{CD}^{+}{ }^{+}$cells) or CD8 (mean, 35.1\%). Peripheral blood cells isolated from the same participants in parallel contained a higher proportion of $\mathrm{CD} 4^{+}$cells $(63.4 \%, P=0.0001)$ and slightly lower proportion of $\mathrm{CD} 8{ }^{+}$cells $(31.8 \%, P=0.005$; Figure 1a), resulting in a substantially reduced $\mathrm{CD} 4 / \mathrm{CD} 8$ ratio in the foreskin compared with blood (1.53 vs. $2.27 ; P<0.0001)$. A small proportion of $\mathrm{CD}^{+}$cells in both the foreskin and peripheral blood were found to express both CD8 and CD4 (0.41\% and 0.84\%, respectively, not significantly different). Of note, the foreskin contained more than twice as many $\mathrm{CD} 4^{-} / \mathrm{CD}^{-}$(double negative) $\mathrm{CD}^{+}$cells as the blood $(12.4 \%$ vs. $5.1 \%, P<0.0001$; Figure 1b).

\section{CCR5 expression and CD4 + Th17 and T-regulatory subsets in the foreskin}

The great majority of sexually transmitted viruses use CCR5 as a co-receptor. ${ }^{26}$ Therefore, we examined the expression of CCR5 on CD3/CD4 ${ }^{+} \mathrm{T}$ cells isolated from the foreskin and the blood of study participants (Figure 2a). The proportion of foreskin CD $4^{+} \mathrm{T}$ cells expressing CCR 5 was over four-fold higher than that in blood $(41.7 \%$ in the foreskin vs. $9.9 \%$ in peripheral blood mononuclear cells (PBMCs), $P<0.0001$; Figure 2b).

Th17 cells may be preferentially infected by HIV ${ }^{18}$ and the ratio of mucosal Th17/Treg cells is important in HIV immunopathogenesis. ${ }^{27}$ Tregs were defined as $\mathrm{CD} 3{ }^{+} / \mathrm{CD} 4^{+}$cells that
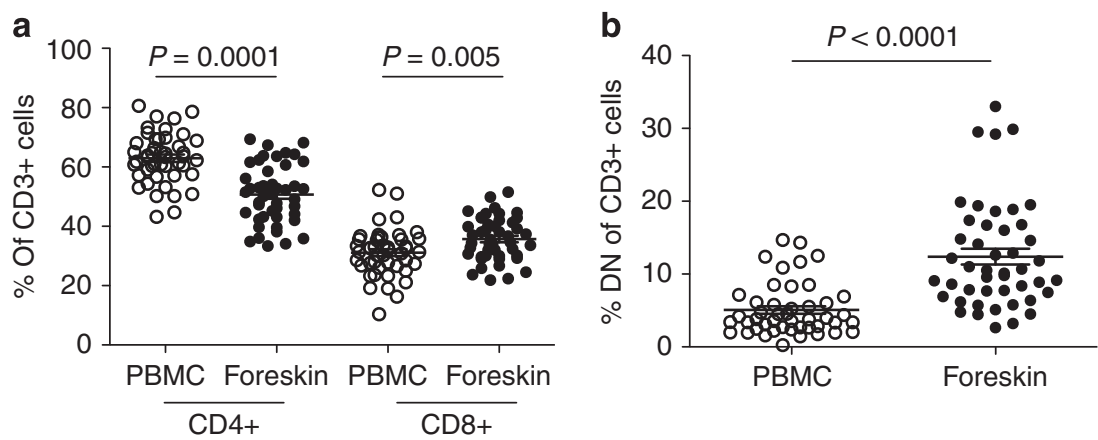

Figure $1 \mathrm{CD}^{+}$and CD8 ${ }^{+}$T-cell subsets within the foreskin and peripheral blood. Peripheral blood mononuclear cells (PBMCs) and foreskin cells from 46 men were stained with CD3-FITC, CD4-PE, and CD8-PerCP. Graphs show percentages of CD3 ${ }^{+}$cells within PBMCs or foreskin cells that co-express (a) either CD4 or CD8, or (b) expressed neither CD4 nor CD8 (double-negative, DN, T cells). 

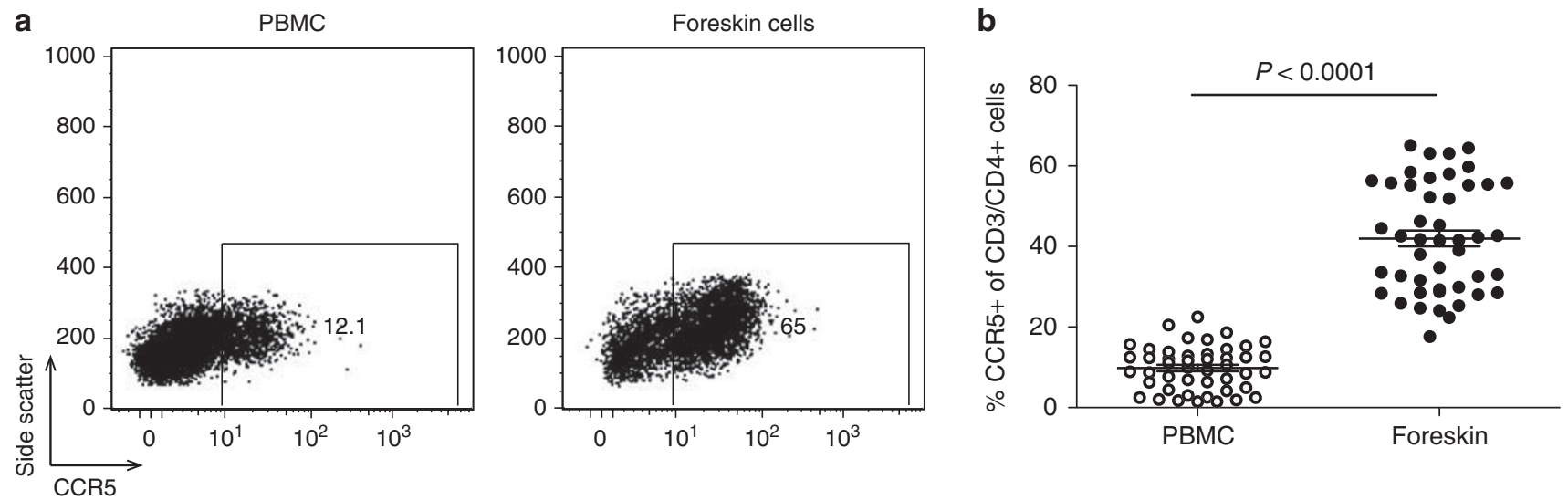

Figure 2 CCR5 expression on CD4 ${ }^{+} \mathrm{T}$ cells from the foreskin and peripheral blood. Peripheral blood mononuclear cells (PBMCs) and foreskin cells from 46 men were stained with CD3-APC, CD4-PE, and CCR5-FITC. Plots in panel a were created by gating on CD3 ${ }^{+} / \mathrm{CD}^{+}{ }^{+}$events. The gate defining $\mathrm{CCR}^{+}$events was created based on PBMC staining for this marker and applied to foreskin plots. (b) Proportions of CD3 ${ }^{+} / \mathrm{CD} 4^{+}$cells in PBMCs and foreskin cells co-expressing CCR5.
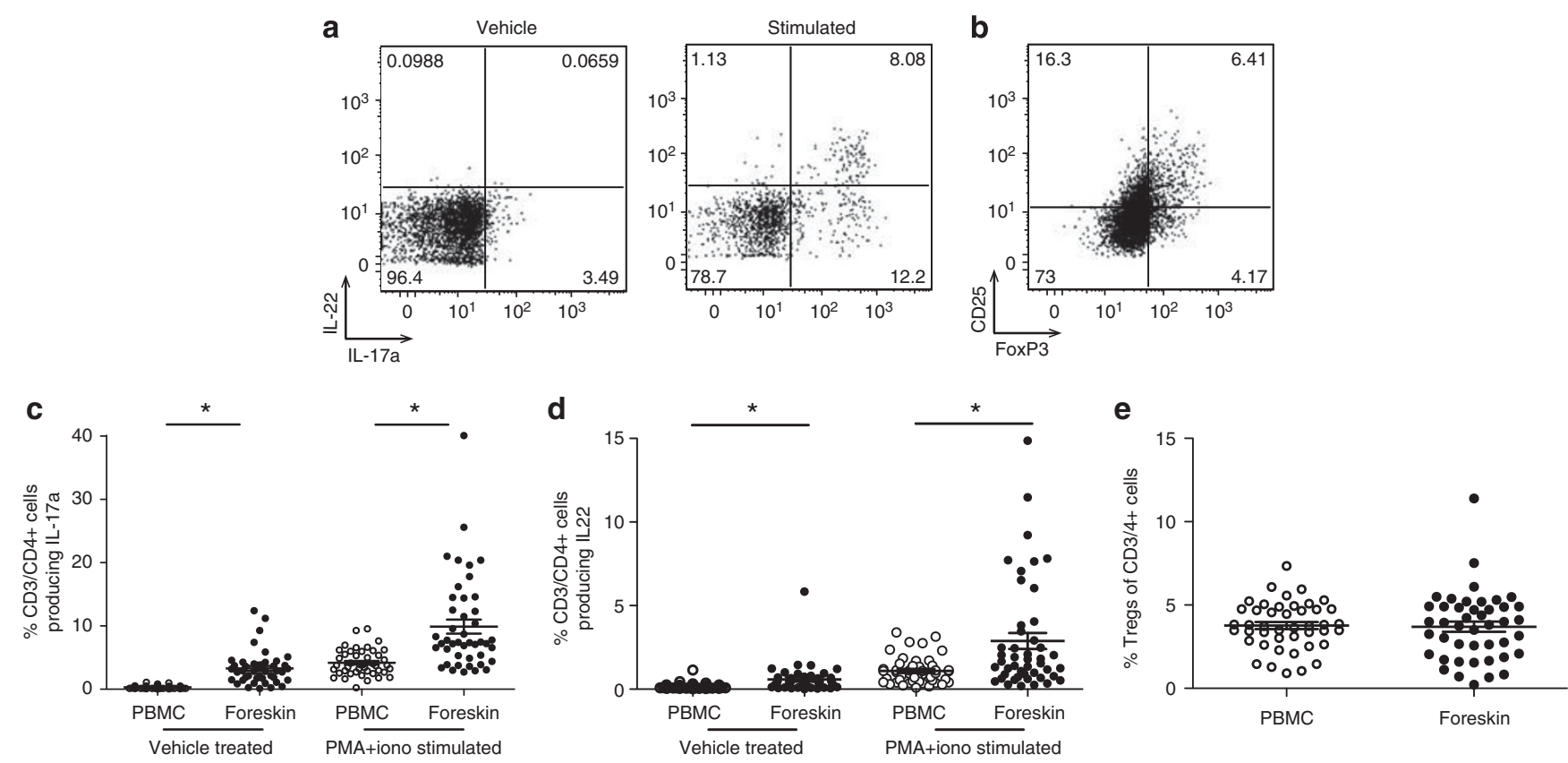

Figure 3 Increased production of interleukin (IL)-17a and IL-22 by foreskin CD4 ${ }^{+} \mathrm{T}$ cells, with no increase in T-regulatory (Treg) frequency. Peripheral blood mononuclear cells (PBMCs) and foreskin cells from 46 men were either left unstimulated (vehicle/Treg) or treated with phorbol-12-myristate13-acetate (PMA)-ionomycin (stimulated). Representative plots of foreskin cells are shown (a, b). The gates in panel a defining IL-17a+ ${ }^{+}$and IL-22+ events were created based on unstimulated PBMC staining for each patient, and then applied to stimulated PBMC and foreskin plots. The gate defining CD25 $5^{+}$events in panel $\mathbf{b}$ was created based on CD25-FMOs (fluorescence minus one = CD3, CD4, and FoxP3). (c) Proportions of Th17 cells in PBMC and foreskin samples $\left(\mathrm{CD}^{+} / \mathrm{CD}^{+} / \mathrm{IL}_{17 \mathrm{a}^{+}}\right.$). (d) IL-22 production in stimulated PBMC and foreskin CD4T cells. (e) Proportions of PBMC and foreskin $\mathrm{CD}^{+} / \mathrm{CD} 4^{+}$cells that are Tregs. ${ }^{*} P<0.0001$.

co-expressed CD25 and the transcription factor FoxP3, and Th17 cells were defined as CD4 ${ }^{+}$T cells producing IL-17a, either at rest or upon stimulation with phorbol-12-myristate-13-acetate-ionomycin. Substantial differences in Th17 subsets were seen between foreskin and blood. Specifically, a much higher proportion of isolated foreskin $\mathrm{CD}^{+} \mathrm{T}$ cells produced IL- $17 \mathrm{a}$, both unstimulated $\left(3.3 \%\right.$ of $\mathrm{CD} 3 / 4^{+}$cells vs. $0.30 \%, P<0.0001)$ and after stimulation $(7.4 \%$ vs. $3.8 \%$, $P<0.0001$; Figure 3a and c). In addition, a higher proportion of $\mathrm{CD}^{+}{ }^{+} \mathrm{T}$ cells from the foreskin produced the Th17-associated cytokine IL-22 than in the peripheral blood, both at rest $(0.166 \%$ vs. $0.579 \% ; P<0.0001)$ and after stimulation $(1.09 \%$ vs. $2.88 \%, P<0.0001$; Figure $3 \mathbf{a}$ and d). However, no difference was observed in the frequency of Tregs between the foreskin and blood (3.9\% of foreskin $\mathrm{CD} 3 / 4^{+}$cells vs. $3.7 \%$ in blood; Figure $3 \mathbf{b}$ and $\mathbf{e})$. As a consequence, the Th17/Treg ratio was considerably higher in the foreskin that in the blood $(4.1 \% \mathrm{vs}$. $1.3 \%$, respectively; $P<0.0001)$. 

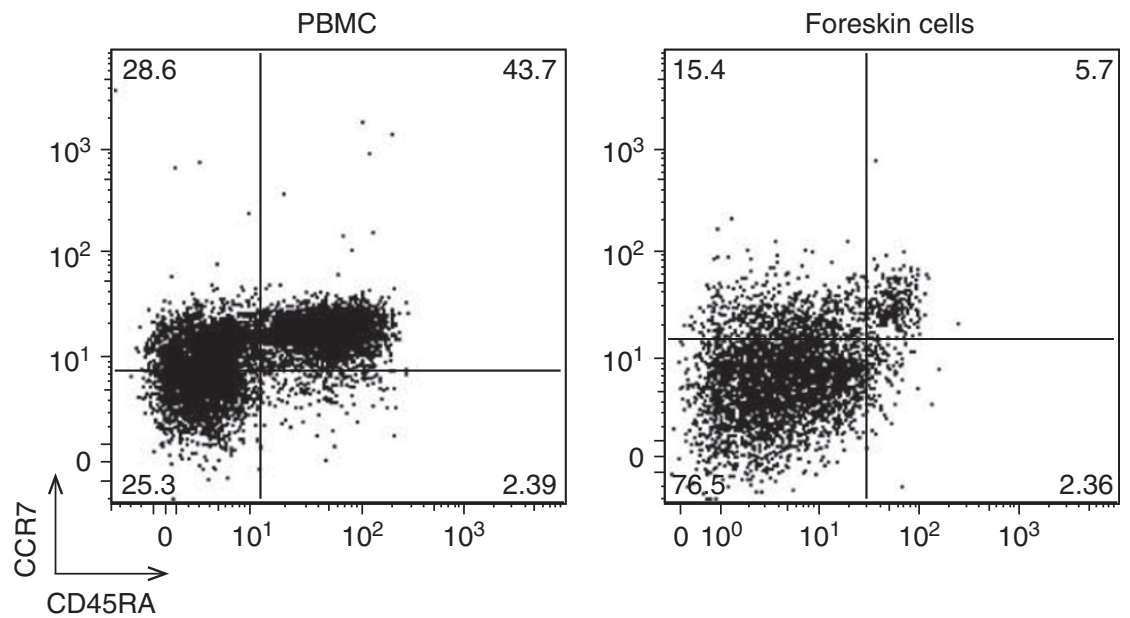

Figure 4 The foreskin contains primarily effector memory CD4 T cells $\left(T_{E M}\right)$. Memory phenotype of peripheral blood mononuclear cells (PBMC) and foreskin mononuclear cells was assessed on a subgroup of three men by staining with CD3-FITC, CD4-PerCP, CCR7-PE, and CD45RA-APC. Representative plots were created by gating on $\mathrm{CD}^{+} / \mathrm{CD} 4^{+}$events. The gates defining $\mathrm{CD} 45 \mathrm{RA}^{+}$and $\mathrm{CCR} 7^{+}$events were created based on $\mathrm{FMO}$ (fluorescence minus one) staining for each cell type.

\section{Foreskin $\mathrm{CD4}{ }^{+} \mathrm{T}$ cells display a predominantly effector memory phenotype}

The memory phenotype of foreskin and blood CD $4^{+} \mathrm{T}$ cells was assessed in a subset of three individuals (representative plots; Figure 4) by staining with CD45RA to distinguish naive $\left(\mathrm{CD} 45 \mathrm{RA}^{+}\right)$from memory $\mathrm{T}$ cells $\left(\mathrm{CD} 45 \mathrm{RA}^{-}\right)$, and CCR7 to further delineate central $\left(\mathrm{T}_{\mathrm{CM}} ; \mathrm{CD} 45 \mathrm{RA}^{-} / \mathrm{CCR} 7^{+}\right)$ and effector $\left(\mathrm{T}_{\mathrm{EM}}\right.$; $\left.\mathrm{CD} 45 \mathrm{RA}^{-} / \mathrm{CCR}^{-}\right)$memory cells. ${ }^{28}$ While blood contained approximately equal proportions of naive and memory $\mathrm{T}$ cells, the foreskin contained few naive $\mathrm{T}$ cells (ranging from 1.2 to $5.8 \%$ ). Of the memory $\mathrm{CD}^{+}$ $\mathrm{T}$ cells in the foreskin, the majority were of the $\mathrm{T}_{\mathrm{EM}}$ phenotype (72.6-89.5\%).

\section{Capacity of foreskin T cells to produce proinflammatory cytokines}

As an inflammatory mucosal immune milieu may enhance HIV acquisition, ${ }^{11}$ we next assessed the production of the cytokines interferon (IFN) $\gamma$ and TNF $\alpha$ by CD8 ${ }^{+}$and $\mathrm{CD} 8^{-}$T-cell subsets, both at rest and after stimulation (Figure 5a). A relatively low frequency of foreskin $\mathrm{T}$ cells produced proinflammatory cytokines prior to stimulation, but this frequency was higher than blood T cells for both TNF $\alpha$ ( $2.8 \%$ vs. $0.47 \%, P<0.0001$; Figure 5b) and IFN $\gamma(0.33 \%$ vs. $0.19 \%, P=0.048$; Figure 5c). Similarly, a higher frequency of foreskin $\mathrm{CD} 8^{+} \mathrm{T}$ cells produced proinflammatory cytokines after mitogen stimulation: this was the case for both TNF $\alpha$ ( $45.3 \%$ vs. $39.4 \%, P=0.0029$; Figure 5b), IFN $\gamma(48.2 \%$ vs. $41.3 \%, P=0.025$; Figure 5c), and for bifunctional cells coproducing both cytokines (35.2\% vs. $28.6 \%$, $P=0.035$; Figure 5d).

While both peripheral blood and foreskin contained a small proportion of "double-negative" $\left(\mathrm{CD} 4^{-} / \mathrm{CD}^{-}\right) \mathrm{T}$ cells, the great majority of $\mathrm{CD} 3^{+} / \mathrm{CD} 8^{-}$cells were $\mathrm{CD} 4^{+} \mathrm{T}$ cells (Figure 1 ). Therefore, we also quantified TNF $\alpha$ and IFN $\gamma$ production in these $\mathrm{CD} 8^{-} \mathrm{T}$ cells as a proxy for $\mathrm{CD} 4^{+} \mathrm{T}$ cells. A greater frequency of foreskin $\mathrm{CD} 8^{-} \mathrm{T}$ cells than blood produced proinflammatory cytokines prior to stimulation $(0.98 \%$ of foreskin cells produced TNF $\alpha$ vs. $0.29 \%$ of those from blood, $P<0.0001 ; 0.48 \%$ of foreskin cells produced IFN $\gamma$ vs. $0.18 \%$ of blood, $P<0.01)$. After mitogen stimulation, the foreskin contained more CD8 ${ }^{-}$T cells producing IFN $\gamma$ ( $45.1 \%$ of foreskin T cells vs. $40.9 \%$ of blood T cells, $P=0.0006)$ and more bifunctional cells $(20.6 \%$ vs. $15.9 \%, P<0.0027)$, although no difference in the frequency of cells producing TNF $\alpha$ was apparent between compartments.

\section{DISCUSSION}

While circumcision reduces the incidence of HIV by up to $60 \%$ in heterosexual African men, ${ }^{2-4}$ providing strong evidence that the foreskin is the main site of male HIV acquisition during vaginal sex, ${ }^{29}$ the immunobiology of HIV acquisition in the foreskin is poorly understood. Previous studies of genital immunology, as it relates to the sexual acquisition of HIV, have focused on the female genital tract and gut, as samples are more easily obtained from these sites. ${ }^{30,31}$ While results of the recent circumcision trials have focused interest on the foreskin, immunology studies have often used cadaveric or fixed/cryopreserved tissues, precluding functional immune studies. ${ }^{14,32-37}$ In collaboration with a clinical site providing safe and free male circumcision as an HIV prevention tool, ${ }^{4}$ we have developed field techniques utilizing expedited tissue processing and use of collagenase I for tissue digestion to isolate viable $\mathrm{T}$ cells from foreskin tissue with retention of the expression of T-cell markers and the functional ability to produce multiple cytokines. This has allowed for the characterization of functional foreskin T-cell subsets.

Several clear differences were evident between foreskin and blood T-cell subsets, both in terms of proportions, expression of HIV co-receptor CCR5, memory phenotypes, and the production of proinflammatory cytokines. There was a relative 
a

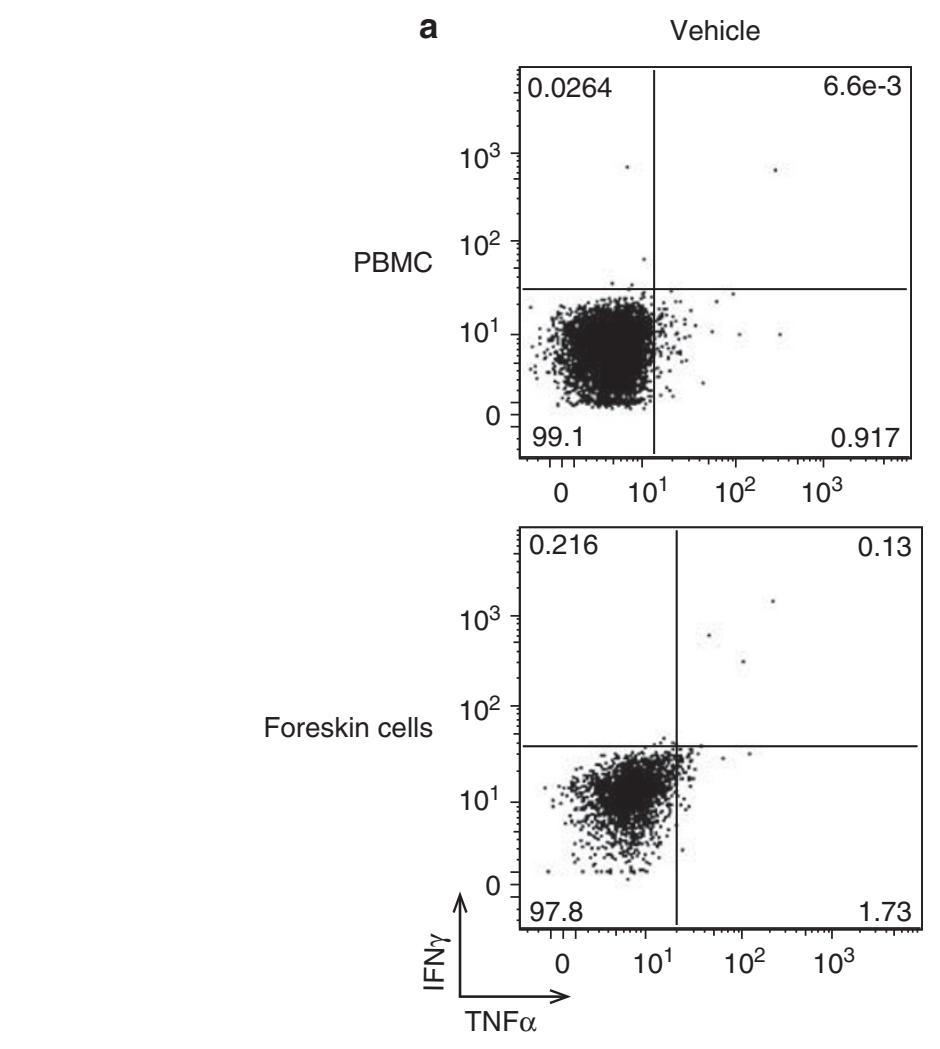

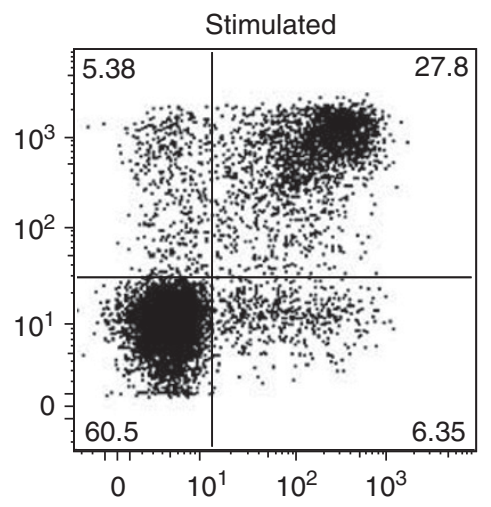

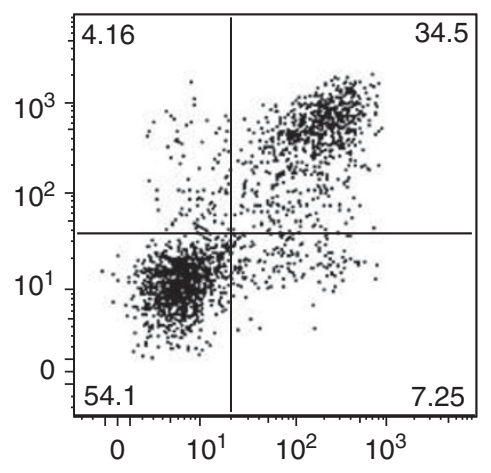

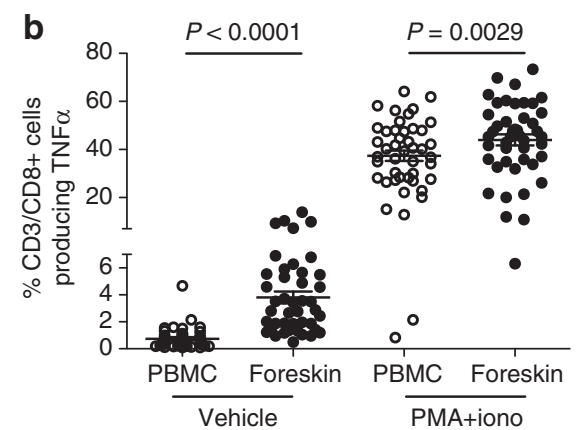
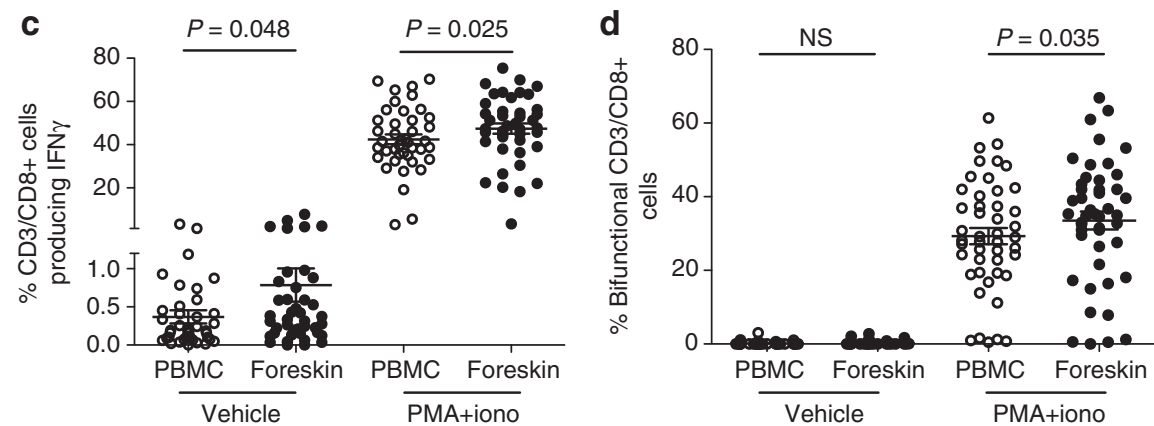

Figure 5 Enhanced production of proinflammatory cytokines by foreskin CD8 ${ }^{+} \mathrm{T}$ cells. Peripheral blood mononuclear cells (PBMCs) and foreskin cells from 46 men were either left unstimulated (vehicle) or treated with phorbol-12-myristate-13-acetate (PMA)-ionomycin. Cells were then stained with CD3-FITC, CD8-PerCP, TNF $\alpha$-PE, and IFN $\gamma$-APC. Plots in panel a were created by gating on $\mathrm{CD}^{+} / \mathrm{CD} 8^{+}$events. The gates defining TNF $\alpha^{+}$ and IFN $\gamma^{+}$events were created based on unstimulated PBMC staining for each patient, and then applied to stimulated PBMC and foreskin plots. (b) Proportions of CD8 T cells producing TNF $\alpha$, (c) IFN $\gamma$, and (d) of bifunctional CD8T cells (producing both TNF $\alpha$ and IFN $\gamma$ ). IFN, interferon; NS, nonsignificant; TNF, tumor necrosis factor.

enrichment of $\mathrm{CD} 8{ }^{+} \mathrm{T}$ cells in the foreskin compared with the blood, contributing to a significantly reduced $\mathrm{CD} 4 / \mathrm{CD} 8$ ratio in the foreskin. However, although the proportion of $\mathrm{CD} 4^{+}$ $\mathrm{T}$ cells was reduced, the proportion of $\mathrm{CD} 4 \mathrm{~T}$ cells in the foreskin that co-expressed CCR5 was over four times higher than in blood, potentially enhancing susceptibility to HIV infection. HIV strains that use CCR5 as an entry co-receptor (R5 strains) are almost always responsible for sexual HIV transmission in vivo, ${ }^{26}$ and an ex vivo model has demonstrated that the foreskin is susceptible to infection with R5-tropic but not X4-tropic viruses. ${ }^{38,39}$ These results indicate that the substantial enhancement of CCR5 expression on foreskin-derived T cells may have direct implications for HIV acquisition.
Interestingly, the proportion of double-negative $\mathrm{T}$ cells (i.e., $\mathrm{CD}^{+}$but $\mathrm{CD}^{-} / \mathrm{CD}^{-}$) was twice as high in the foreskin than in blood. Various $\mathrm{CD}^{+}$T-cell populations may be contained within this subset, including natural killer $\mathrm{T}$ cells ${ }^{40}$ and $\mathrm{T}$ cells bearing the variant $\mathrm{T}$-cell receptors $\gamma \delta^{41}$ or the regulatory TCR $\alpha \beta^{+}{ }^{42}$ Double-negative CD3 ${ }^{+} \mathrm{T}$ cells have been associated with protection against SIV immunopathogenesis in some primate species. ${ }^{43}$ Elucidating the identity of these double-negative cells using multiparameter flow cytometry and investigating their possible relevance for HIV transmission will be important areas for future study.

While phenotypic characterization of foreskin $\mathrm{T}$ cells has been possible using fixed or cryopreserved tissues, we were 
particularly interested to define their function directly ex vivo. Two $\mathrm{CD}^{+} \mathrm{T}$-cell subsets that may be particularly relevant to HIV acquisition and pathogenesis are Th17 and Tregs. ${ }^{27}$ Th17 cells are $\mathrm{CD} 4^{+} \mathrm{T}$ cells producing the cytokine IL-17a, and have a prominent proinflammatory role in mucosal immune defense against invading bacterial and fungal pathogens through the IL-17-mediated recruitment of neutrophils, induction of antimicrobial peptides, and maintenance of epithelial integrity ${ }^{44}$ Th17 cells display enhanced susceptibility to HIV in vitro ${ }^{18,45}$ and are preferentially depleted from the blood and particularly the mucosa of HIV-infected individuals, ${ }^{18,30}$ suggesting their enrichment at mucosal surfaces might enhance HIV acquisition. Tregs have immunomodulatory effects that are thought to have an important role in counterbalancing Th17-induced inflammation, despite sharing a common precursor, chemokine receptors, and mucosal homing properties with Th17 cells. ${ }^{27}$ We found that Th17 proportions were substantially increased in the foreskin compared with blood in the absence of any corresponding enrichment in Treg cells. This increased Th17/Treg ratio in the foreskin suggests that this tissue is biased toward a predominantly proinflammatory immune environment, which could enhance HIV acquisition. ${ }^{11}$

Our data showing enhanced production of the cytokines TNF $\alpha$ and IFN $\gamma$ by foreskin T cells, both at rest and after nonspecific stimulation, support the concept of the foreskin as a proinflammatory tissue. This enhanced cytokine production is likely to be related to the high proportion of effector memory $\mathrm{T}$ cells $\left(\mathrm{T}_{\mathrm{EM}}\right)$ found in the foreskin tissues, as this cell subset is primed to migrate to tissue sites and to carry out immediate effector functions. ${ }^{28}$

It is likely that both the function and proportions of T-cell subsets in the foreskin would be impacted by common bacterial and viral genital coinfections, ${ }^{14,15}$ and any such differences might well have implications for HIV susceptibility. While men with symptomatic genital infections were excluded from male circumcision due to the potential increased risk of post-surgical infection and other complications, asymptomatic genital infections such as HSV-2 and HPV are common in these men. ${ }^{46,47}$ The purpose of our initial analysis was to compare T-cell subsets in the foreskin and blood, but recruitment of a larger participant sample size is ongoing with the goal of characterizing the immune impact of these infections.

While our study examined pooled $\mathrm{T}$ lymphocytes derived from both the inner and outer foreskin, there is in vitro evidence to suggest that HIV acquisition may be more efficient across the inner surface of the foreskin, ${ }^{48}$ defined as the portion of the foreskin that sits against the glans on the non-erect penis, but is exposed on the erect penis during intercourse. It was initially assumed that this increased susceptibility was due to a thinner keratin layer on the inner foreskin, ${ }^{36,48}$ but studies using freshly processed foreskin samples have shown no difference in this layer between the inner and outer foreskin. ${ }^{34,37}$ Although reports of differences in the density of HIV target cells between these two sites have been contradictory, ${ }^{35,36,38,39,49}$ it does seem that cells of the inner foreskin may be functionally different to those of the outer foreskin, both in their responsiveness to cytokines such as TNF $\alpha$ and MIP $1 \alpha^{49}$ and in their production of chemokines after HIV exposure. ${ }^{48-50}$ Better elucidation of the functional differences between $\mathrm{T}$ cells derived from the inner and outer foreskin will constitute an important area for future research.

In summary, we have developed novel techniques to purify a single-cell suspension from fresh foreskin tissues, and to characterize the functional characteristics of foreskin T-cell populations. Compared with blood, the foreskin manifested a proinflammatory immune environment that was enriched for highly HIV-susceptible CD4 ${ }^{+}$T-cell subsets, such as Th17 cells and those expressing the HIV co-receptor CCR5. These observations have important implications for HIV susceptibility in the foreskin, and will inform larger immuno-epidemiology field studies aiming to define the immune correlates of HIV susceptibility in the foreskin.

\section{METHODS}

Participants. Participants were men recruited from an established community cohort in Rakai, Uganda, ${ }^{25}$ who had elected to undergo adult circumcision at the Rakai Health Sciences Program in Kalisizo, Uganda. Foreskins and whole blood samples were obtained from 46 HIV-negative men between the ages of 15 and 49 years. All participants provided written informed consent, and ethical approval was obtained through the research ethics boards of collaborating institutions (the University of Toronto, Uganda Virus Research Institute, and Western IRB). Surgery was deferred in the context of urethral discharge or clinically apparent genital ulceration. Participants were confirmed to be antibody-negative for HIV-1 and HIV-2 using two enzyme-linked immunosorbent assays (Murex HIV-1.2.O, Abbott, Abbott Park, IL; and Vironistika HIV Uni-Form II plus O Mircoelisa System, bioMerieux; Marcy l'Etoile, France). Discordant results were confirmed by western blot (GS HIV-1 Western Blot, BioRad, Hercules, CA). All participants were also screened for acute HIV infection by real-time polymerase chain reaction. RNA was extracted from plasma samples using the Sample Preparation System (Abbott), and amplification was performed using the Real Time HIV-1 Amplification Reagent Kit (Abbott) run on the M2000rt (Abbott).

T-cell isolation from the foreskin and blood. Foreskins were collected into RPMI 1640 media supplemented with: 10\% heat-inactivated fetal bovine serum, $10 \mathrm{U} \mathrm{ml}^{-1}$ penicillin, $10 \mu \mathrm{g} \mathrm{ml}^{-1}$ streptomycin, $250 \mathrm{ng} \mathrm{ml}^{-1}$ amphotericin B, and $2 \mathrm{mM}$ L-glutamine (all from Gibco, Invitrogen, Carlsbad, CA; henceforth referred to as R10 medium). Foreskin samples were always processed within $15 \mathrm{~min}$ of surgery, as additional time caused the dermal morphology to change substantially, with gross macroscopic tissue edema (data not shown). Tissue was first sectioned into longitudinal strips including both inner and outer foreskin and containing both epidermal and dermal tissue. These strips were then further sectioned to create pieces of approximately $0.25 \mathrm{~cm}^{2}$. Each piece was placed in a $1.5-\mathrm{ml}$ conical tube containing $1.0 \mathrm{ml}$ of $500 \mathrm{U} \mathrm{ml}^{-1}$ Collagenase Type I (Gibco) and $42.5 \mathrm{U} \mathrm{ml}^{-1}$ of DNAse (Invitrogen) in RPMI 1640 media supplemented with $10 \mathrm{U} \mathrm{ml}^{-1}$ penicillin, $10 \mu \mathrm{g} \mathrm{ml}^{-1}$ streptomycin, $250 \mathrm{ng} \mathrm{ml}^{-1}$ amphotericin B, and $2 \mathrm{mM} \mathrm{L}$-glutamine (henceforth referred to as RPMI, all from Gibco). Initial immune studies have used dispase for foreskin tissue digestion, but we found that treatment with as little as $1.0 \mathrm{U} \mathrm{ml}^{-1}$ of dispase (Gibco) for $30 \mathrm{~min}$ at $37^{\circ} \mathrm{C}$ led to the loss of $\mathrm{CD} 4$ expression and decreased CD8 expression in both peripheral blood and foreskinderived T cells (Supplementary Figure S1 online). Scissors were used to mechanically disrupt each piece of tissue, and tubes were then placed on a shaker (Eppendorf Thermomixer; Hamburg, Germany) for $30 \mathrm{~min}$ of enzymatic digestion at $37^{\circ} \mathrm{C}$ with shaking at 900 r.p.m. The cellular suspension obtained from each tube was pooled and collagenase activity 
was inhibited by the addition of fetal bovine serum to a final concentration of $10 \%$. This cell suspension was then filtered through a $100-\mu \mathrm{m}$ cell strainer (BD Biosciences, Franklin Lakes, NJ,) to remove any remaining undigested tissue. Filtered cells were washed once, resuspended in R10, and allowed to rest under normal growth conditions $\left(37^{\circ} \mathrm{C} 5 \% \mathrm{CO}_{2}\right.$, humidified atmosphere) for 3-7h. This combination of collagenase 1 and gentle mechanical digestion allowed for the retention of CD4 expression and gave a single-cell suspension containing $\mathrm{CD} 3^{+} \mathrm{T}$ cells that showed a similar CD4 and CD8 expression profile to PBMCs from the same individual (Supplementary Figure S2 online).

PBMCs were isolated by density gradient centrifugation (Ficoll-Paque Plus; Amersham Biosciences, Uppsala, Sweden).

Characterization of $\mathrm{CD4}^{+}$T-cell subsets. Both PBMC and foreskin cell numbers were determined by trypan blue exclusion. PBMCs $\left(1 \times 10^{6}\right)$ and foreskin $\left(10\right.$ to $\left.20 \times 10^{6}\right)$ cells (depending on yield) were plated in $500 \mu \mathrm{l} \mathrm{R} 10$ and stimulated with either $1 \mathrm{ng} \mathrm{ml}^{-1}$ phorbol-12-myristate13-acetate and $1 \mu \mathrm{g} \mathrm{ml}^{-1}$ ionomycin (both from Sigma, St Lousi, MO) or vehicle ( $0.1 \%$ dimethylsulfoxide) with $5 \mu \mathrm{g} \mathrm{ml}^{-1}$ Brefeldin A (GolgiPlug, BD Biosciences) for $9 \mathrm{~h}$ at $37^{\circ} \mathrm{C}$. Samples were then washed with cold $2 \%$ fetal bovine serum in phosphate-buffered saline and stained with fluorochrome-labeled monoclonal antibodies specific for CD3 (UCHT1), CD4 (RPA-T4), CD8 (SK1), CCR5 (2D7/CCR5), and CD25 (M-A251; all BD Biosciences). Excess surface antibody was removed by washing with $2 \%$ fetal bovine serum in phosphate-buffered saline. Samples for intracellular staining were permeabilized using either the eBioscience fixation/ permeabilization solution for Treg identification (eBiosciences, San Diego, CA) or the BD Cytofix/Cytoperm solution (BD Biosciences) for all others. Cells were washed in permeabilization wash buffer and stained with fluorochrome-labeled monoclonal antibodies specific for combinations of the following intracellular cytokines/transcription factors: TNF $\alpha$ (MAb11; BD Biosciences), IFN $\gamma$ (B27; BD Biosciences), IL-17a (eBio64DEC17; eBioscience), IL-22 (22URTI; eBioscience), and FoxP3 (PCH101; eBioscience). Samples were acquired using a FACSCalibur flow cytometer (BD Systems) and data analysis performed using FlowJo analytical software version v.9.3 (Treestar, Ashland, OR).

Unpermeabilized foreskin cells were gated based on forward and sideward scatter (Supplementary Figure S2 online). This gate was created based on the location of $\mathrm{CD}^{+} \mathrm{T}$ cells in the PBMC sample from the same patient. Back gating was used to confirm that this gate corresponded with the location of $\mathrm{CD}^{+}$cells in the foreskin sample $\left(\mathrm{CD}^{+}\right.$cells representing approximately $0.1-0.6 \%$ of total events in the foreskin sample). A clearly visible population of $\mathrm{CD}^{+}$cells in the foreskin samples could then be identified. For unpermeabilized foreskin cells, $10^{6}$ events were recorded, while only $10^{5}$ events were recorded for PBMC and permeabilized foreskin cells, due to the large amount of other cell types present in unpermeabilized foreskin samples. After permeabilization the T-cell population was enriched (3-5\% of total events) and could be directly identified on the forward by side scatter plot (Supplementary Figure S2 online).

Statistical analysis. T-cell populations were compared between blood and foreskin by paired Wilcoxon rank-sum test. Statistical tests were run on SPSS v.17.0 for Mac (IBM; New York, NY). Flow cytometry data were analyzed in FlowJo v.9.3 and Excel (Microsoft; Redmond, WA) prior to statistical testing.

SUPPLEMENTARY MATERIAL is linked to the online version of the paper at http://www.nature.com/mi

\section{ACKNOWLEDGMENTS}

We acknowledge the support of the Rakai Health Sciences staff and thank the men who participated in this study. This study was supported by the Ontario HIV Treatment Network (J.L.P., studentship); the Canada Research Chair Programme (R.K., salary support); the Bill and Melinda Gates Foundation (R.G., \#22006.02); the Canadian Institutes of Health Research (R.K., \# HBF115704); and the National Institute of Health (R.G., R01A1087409-02).

\section{DISCLOSURE}

The authors declared no conflict of interest.

2012 Society for Mucosal Immunology

\section{REFERENCES}

1. UNAIDS Global report: UNAIDS report on the global AIDS epidemic 2010 1-364 (2010)

2. Auvert, B. et al. Randomized, controlled intervention trial of male circumcision for reduction of HIV infection risk: the ANRS 1265 trial. PLoS Med. 2, e298 (2005).

3. Bailey, R. et al. Male circumcision for HIV prevention in young men in Kisumu, Kenya: a randomised controlled trial. The Lancet 369, 643-656 (2007).

4. Gray, R.H. et al. Male circumcision for HIV prevention in men in Rakai, Uganda: a randomised trial. The Lancet 369, 657-666 (2007).

5. Anderson, D., Politch, J.A. \& Pudney, J. HIV infection and immune defense of the penis. Am. J. Reprod. Immunol. 65, 220-229 (2011).

6. Kigozi, G. et al. Foreskin surface area and HIV acquisition in Rakai, Uganda (size matters). AIDS 23, 2209-2213 (2009).

7. Ganor, Y. \& Bomsel, M. HIV-1 transmission in the male genital tract. Am J Reprod Immunol 65, 284-291 (2011).

8. de Bruyn, G. et al. Uptake of male circumcision in an HIV vaccine efficacy trial. J. Acquir. Immune Defic. Syndr. 51, 108-110 (2009).

9. Li, Q. et al. Glycerol monolaurate prevents mucosal SIV transmission. Nature 458, 1034-1038 (2009).

10. Zhang, Z. Sexual transmission and propagation of SIV and HIV in resting and activated CD4+ T cells. Science 286, 1353-1357 (1999).

11. Kaul, R. et al. The genital tract immune milieu: an important determinant of HIV susceptibility and secondary transmission. J. Reprod. Immunol. 77, 32-40 (2008).

12. Zhang, Z.Q. Roles of substrate availability and infection of resting and activated CD4+ T cells in transmission and acute simian immunodeficiency virus infection. Proc. Natl. Acad. Sci. 101, 5640-5645 (2004).

13. Haase, A.T. Early events in sexual transmission of HIV and SIV and opportunities for interventions. Annu. Rev. Med. 62, 127-139 (2011).

14. Johnson, K.E. et al. Effects of HIV-1 and herpes simplex virus Type 2 infection on lymphocyte and dendritic cell density in adult foreskins from Rakai, Uganda. J. Infect. Dis. 203, 602-609 (2011).

15. Rebbapragada, A. et al. Negative mucosal synergy between Herpes simplex type 2 and HIV in the female genital tract. AIDS 21, 589-598 (2007).

16. Zhu, J. et al. Persistence of HIV-1 receptor-positive cells after HSV-2 reactivation is a potential mechanism for increased HIV-1 acquisition. Nat. Med. 15, 886-892 (2009).

17. Freeman, E.E. et al. Herpes simplex virus 2 infection increases HIV acquisition in men and women: systematic review and meta-analysis of longitudinal studies. AIDS 20, 73-83 (2006).

18. El Hed, A. et al. Susceptibility of human Th17 cells to human immunodeficiency virus and their perturbation during infection. J. Infect. Dis. 201, 843-854 (2010).

19. McKinnon, L.R. et al. Characterization of a human cervical CD4+ T cell subset coexpressing multiple markers of HIV susceptibility. J. Immunol.; advance online publication, 2 November 2011; doi:10.4049/ jimmunol.1101836 (2011) (e-pub ahead of print).

20. Kim, C.J. \& Kaul, R. Th22 cells constitute a highly HIV susceptible T cell subset that is associated with epithelial integrity in the sigmoid mucosa. in. 20th Annual Canadian Conference on HIVIAIDS Research (CAHR) (Toronto, Ontario, 2011).

21. Card, C.M. et al. Decreased immune activation in resistance to HIV-1 infection is associated with an elevated frequency of CD4+CD25+FOXP3 +regulatory T cells. J. Infect. Dis. 199, 1318-1322 (2009).

22. Jennes, W. et al. Suppressed cellular alloimmune responses in HIVexposed seronegative female sex workers. Clin. Exper. Immunol. 143, 435-444 (2006).

23. McLaren, P.J. et al. HIV-exposed seronegative commercial sex workers show a quiescent phenotype in the CD4+T cell compartment and reduced expression of HIV-dependent host factors. J. Infect. Dis. 202, S339-S344 (2010).

24. Bégaud, E. et al. Reduced CD4 T cell activation and in vitro susceptibility to HIV-1 infection in exposed uninfected Central Africans. Retrovirology 3, 35 (2006). 
25. Gray, R.H. et al. Male circumcision and HIV acquisition and transmission: cohort studies in Rakai, Uganda. AIDS 14, 2371-2381 (2000).

26. Grivel, J.-C., Shattock, R.J. \& Margolis, L.B. Selective transmission of R5 HIV-1 variants: where is the gatekeeper? J. Transl. Med. 9, S6 (2010).

27. Kanwar, B., Favre, D. \& McCune, J.M. Th17 and regulatory T cells: implications for AIDS pathogenesis. Curr. Opin. HIV AIDS 5, 151-157 (2010).

28. Sallusto, F., Lenig, D., Forster, R., Lipp, M. \& Lanzavecchia, A. Two subsets of memory $T$ lymphocytes with distinct homing potentials and effector functions. Nature 401, 708-712 (1999).

29. Dinh, M.H., Fahrbach, K.M. \& Hope, T.J. The role of the foreskin in male circumcision: an evidence-based review. Am. J. Reprod. Immunol. 65, 279-283 (2011).

30. Chege, D. et al. Sigmoid Th17 populations, the HIV latent reservoir, and microbial translocation in men on long-term antiretroviral therapy. AIDS 25, 741-749 (2011).

31. Kaul, R. et al. HIV-1-specific mucosal CD8+ lymphocyte responses in the cervix of HIV-1-resistant prostitutes in Nairobi. J. Immunol. 164, 1602-1611 (2000).

32. Bailey, R.C. et al. HIV-1 target cells in foreskins of African men with varying histories of sexually transmitted infections. Am. J. Clin. Pathol. 125, 386-391 (2006).

33. Hirbod, T. et al. Abundant expression of HIV target cells and C-type lectin receptors in the foreskin tissue of young Kenyan men. Am. J. Pathol. 176, 2798-2805 (2010).

34. Dinh, M.H., McRaven, M.D., Kelley, Z.L., Penugonda, S. \& Hope, T.J. Keratinization of the adult male foreskin and implications for male circumcision. AIDS 24, 899-906 (2010).

35. Hussain, L.A. \& Lehner, T. Comparative investigation of Langerhans' cells and potential receptors for HIV in oral, genitourinary and rectal epithelia. Immunology 85, 475-484 (1995).

36. McCoombe, S.G. \& Short, R.V. Potential HIV-1 target cells in the human penis. AIDS 20, 1491-1495 (2006).

37. Qin, Q. et al. Langerhans' cell density and degree of keratinization in foreskins of Chinese preschool boys and adults. Int. Urol. Nephrol. 41, 747-753 (2009).

38. Fischetti, L., Barry, S.M., Hope, T.J. \& Shattock, R.J. HIV-1 infection of human penile explant tissue and protection by candidate microbicides. AIDS 23, 319-328 (2009).
39. Patterson, B.K. et al. Susceptibility to human immunodeficiency virus-1 infection of human foreskin and cervical tissue grown in explant culture. Am. J. Pathol. 161, 867-873 (2002).

40. Bendelac, A., Savage, P.B. \& Teyton, L. The Biology of NKT Cells. Annu. Rev. Immunol. 25, 297-336 (2007).

41. Gollob, K., Antonelli, L., Faria, D., Keesen, T. \& Dutra, W. Immunoregulatory mechanisms and CD4-CD8- (double negative) T cell subpopulations in human cutaneous leishmaniasis: a balancing act between protection and pathology. Int. Immunopharmacol. 8, 1338-1343 (2008).

42. Zhang, D. et al. New differentiation pathway for double-negative regulatory $T$ cells that regulates the magnitude of immune responses. Blood 109, 4071-4079 (2007).

43. Milush, J.M. et al. Lack of clinical AIDS in SIV-infected sooty mangabeys with significant CD4+ T cell loss is associated with double-negative T cells. J. Clin. Invest. 121, 1102-1110 (2011).

44. O'Connor, W., Zenewicz, L.A. \& Flavell, R.A. The dual nature of TH17 cells: shifting the focus to function. Nat. Immunol. 11, 471-476 (2010).

45. Gosselin, A. et al. Peripheral blood CCR4+CCR6+ and CXCR3+CCR6+ CD4+ T cells are highly permissive to HIV-1 infection. J. Immunol. 184, 1604-1616 (2009).

46. Gray, R.H. et al. Male circumcision decreases acquisition and increases clearance of high-risk human papillomavirus in HIV-negative men: a randomized trial in Rakai, Uganda. J. Infect. Dis. 201, 1455-1462 (2010).

47. Tobian, A.A.R. et al. Factors associated with the prevalence and incidence of herpes simplex virus type 2 infection among men in Rakai, Uganda. J. Infect. Dis. 199, 945-949 (2009).

48. Ganor, Y. et al. Within $1 \mathrm{~h}, \mathrm{HIV}$-1 uses viral synapses to enter efficiently the inner, but not outer, foreskin mucosa and engages Langerhans-T cell conjugates. Mucosal Immunol. 3, 506-522 (2010).

49. Fahrbach, K.M., Barry, S.M., Anderson, M.R. \& Hope, T.J. Enhanced cellular responses and environmental sampling within inner foreskin explants: implications for the foreskin's role in HIV transmission. Mucosal Immunol. 3, 410-418 (2010).

50. Zhou, Z. et al. HIV-1 efficient entry in inner foreskin is mediated by elevated CCL5/RANTES that recruits T cells and fuels conjugate formation with Langerhans cells. PLoS Pathog 7, e1002100 (2011). 